\title{
Infection after the use of INFIX in Pelvic Ring Injuries
}

\author{
Rahul Vaidya*(1), Karun Amar, Derrek Woodbury, and Austen Washington (1) \\ Detroit Medical Center, 5th Floor Heart Hospital, 311 Mack Avenue, Detroit, Michigan 48201, USA
}

Received 14 June 2021, Accepted 11 August 2021, Published online 8 September 2021

\begin{abstract}
Introduction: The purpose of this study is to report on infection with anterior subcutaneous internal pelvic fixation (INFIX) for pelvic ring injuries and the outcomes of treatment. Methods: An IRB-approved retrospective study was performed using trauma databases of a level one and level two trauma center from 2012-2018. Infection after the INFIX procedure was diagnosed in 10 of 179 cases. Treatment included formal irrigation and debridement, removal of the hardware, and culture-specific antibiotics. Patients were followed for a minimum of 12 months. Recorded outcomes include X-rays, Majeed scores, and the presence of any loss of reduction using reduction parameters. Results: Time to detect the infection was $54.2 \pm 24.3$ days (range 24-90, median 56 days). Staphylococcus aureus was the most common bacteria isolated. The average follow-up was $830 \pm 170$ days (range 575-1088 days). All patients went on to the radiographic union. There were no recurrent infections or osteomyelitis at the latest follow-up. Patients maintained their reduction after INFIX removal (KI), and Majeed scores ranged from 72 to 96 (seven good, three excellent). Discussion: Infections after using the INFIX procedure were dealt with by irrigating and debriding the wounds, removing the INFIX with culture-specific antibiotics for 2-6 weeks. Implants were maintained for at least 25 days, and there was no loss of reduction. There were no long-term sequelae noted in this small series or the literature review included in this paper.
\end{abstract}

Key words: Infection, Infix, Irrigation, Debridement, Pelvic, Ring.

\section{Introduction}

The use of an anterior subcutaneous internal fixator (INFIX) has been reported in the literature to reduce and fix unstable pelvic ring injuries with the appropriate posterior fixation [1-19]. Most authors report the INFIX is well tolerated by patients, allowing good mobility and outcomes. The downside is the need for secondary surgery to remove the implants, recommended three months post-op or later [20]. A recent systematic review reports heterotopic ossification (36\%), lateral femoral cutaneous nerve (LFCN) irritation (26.3\%), infection, and femoral nerve palsy (1\%) as some of the possible complications. The incidence of infection after INFIX is reported as $1-3 \%$ [1-19]. Due to the limited numbers of infected cases in the INFIX series, there is no current recommendation on treatment. The purpose of this paper is to report on infections after the use of INFIX for pelvic ring injuries, report on outcomes and review the literature. We also asked if there is a risk of loss of reduction by removing the INFIX early.

\section{Materials and methods}

An IRB-approved retrospective study was performed using trauma databases of one level one and one level two trauma

\footnotetext{
*Corresponding author: rahvaidya2012@gmail. com
}

center. We retrospectively reviewed all patients from July 2012 to December 2018 who underwent INFIX and appropriate posterior fixation. The indication for this index surgery was an unstable pelvic fracture in which the surgeon believed that there was a need for anterior fixation. At the time of index fixation, all the patients underwent supplemental percutaneous sacroiliac screw fixation or open fixation of the posterior pelvis plus lumbopelvic fixation when indicated. Per the Surgical Care Improvement Project guidelines, all patients received preoperative antibiotics before the start of surgery.

Among this group of patients, 10 of 179 (5.6\%) were subsequently diagnosed with infection and were included in this study. There were five women and five men with an average age of 37.4 years (range, 14-67 years) and a follow-up of $830 \pm 170$ days (range 575-1088 days). Two of ten infected cases also had supplemental lumbopelvic fixation.

The following information was recorded: age, gender, presence of comorbidities, Young/Burgess fracture classifications [21], date of initial surgery, date of implant removal, length of follow-up, method of injury, associated injuries, and culture-specific antibiotic. (Table 1) Positive cultures from a draining wound determined presence of infection. Upon detection, patients were taken to the operating room for formal irrigation and debridement (I\&D) and hardware removal. Irrigation and debridement was performed with deep cultures taken from 
Table 1. Patient demographics.

\begin{tabular}{|c|c|c|c|c|c|c|c|c|}
\hline Patient & Age & Sex & BMI & Class & Other pelvic fixation & Bug isolated & $\begin{array}{c}\text { \# of } \\
\text { washout }\end{array}$ & $\begin{array}{c}\text { follow-up } \\
\text { days } \\
\end{array}$ \\
\hline 1 & 26 & $\mathrm{~F}$ & 21.7 & LC3 & $\mathrm{R}$ S2 SI $\times 2$ & Strep. agalactiae & 1 & 783 \\
\hline 2 & 45 & M & 27.2 & $\mathrm{LC} 1$ & R S1 SI, R S2 SI & MSSA & 1 & 975 \\
\hline 3 & 67 & M & 22.4 & LC3 & R S1 SI, L S1 SI & Enterobacter cloacae & 2 & 977 \\
\hline 4 & 57 & M & 17 & $\mathrm{LC} 1$ & $\begin{array}{l}\text { R S1 SI, L S1 SI } \\
\text { Bilat lumbopelvic } \\
\text { fixation }\end{array}$ & MSSA & 1 & 1088 \\
\hline 5 & 53 & $\mathrm{~F}$ & 36 & $\mathrm{LC} 2$ & R S1 SI, R S2 SI, Plates & $\begin{array}{c}\text { Corynebacterium, Staphylococcus } \\
\text { epidermidis, Proteus mirabilis }\end{array}$ & 1 & 840 \\
\hline 6 & 26 & M & 25.2 & $\mathrm{LC} 2$ & R S1 SI & MRSA & 1 & 905 \\
\hline 7 & 21 & $\mathrm{~F}$ & 24.7 & LC 3 & R Lumbopelvic, R S1 TSS & MSSA & 1 & 881 \\
\hline 8 & 28 & $\mathrm{~F}$ & 24.3 & APC2/APC3 & Left S1 SI, S2 TSS & MRSA & 2 & 605 \\
\hline 9 & 14 & $\mathrm{M}$ & 42.3 & APC3/APC2 & Bilateral S1 SI & Enterobacter cloacae & 2 & 575 \\
\hline 10 & 87 & $\mathrm{~F}$ & 24 & $\mathrm{LC} 2$ & L 2 LC2 Screws & MSSA & 1 & 672 \\
\hline
\end{tabular}

S1 or S2 = sacral body segment; SI, sacroiliac screw; TSS, Trans sacral screw; LC2 = Lateral compression two screw; MRSA, methicillinresistant Staphylococcus aureus; MSSA, methicillin-sensitive Staphylococcus aureus; RS1 SI = Right Sacral 1st body sacroiliac screw.

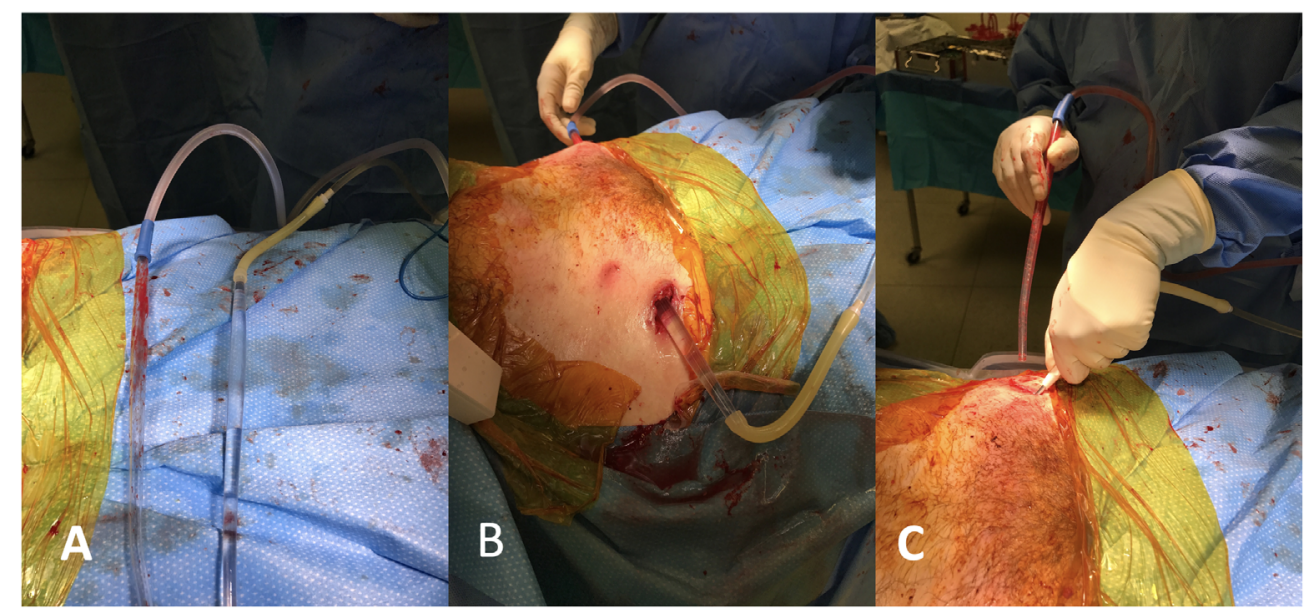

Figure 1. (a) After removing the implants, the wounds were washed with 6-9 L of Normal Saline with cystoscopy tubing and a plastic Yankour tip. (b) For the subcutaneous tunnel of the bar, on one side, the saline flowed in, and on the other, we used suction. (c) The pin sites were also curetted in the bone and washed with smaller tips fastened to the cystoscopy tubing.

pin sites and the area of the subcutaneous bar. The skin incisions were not expanded from the original insertion procedure. We washed with 6-9 L of Normal Saline with cystoscopy tubing and a Yankauer tip (Figure 1), curetted the pin sites, and removed the INFIX. The Yankauer suctions were used to instill irrigation and suction it out from the subcutaneous tunnel (Refer Supplementary Video). After the I\&D, culture-specific antibiotics were recommended and administered by the infectious disease team with close follow-up in the clinic. Standard pelvic imaging series consisting of anterior posterior (AP) and inlet/ outlet views were obtained in the clinic, and the radiographic union was assessed. Patients were followed for a minimum of 12 months in the clinic with X-rays. Patients were invited back for a final visit for this study where an exam, X-rays, and clinical outcome score as described by Majeed [22] was performed. Loss of reduction was measured using the modified Keshishyan Index [23, 24].

\section{Results}

The infection rate was $10 / 179(5.6 \%)$, which is slightly higher than reported in the literature (see Table 3). Risk factors included: One patient with insulin-dependent diabetes mellitus, one had hepatitis $\mathrm{C}$ liver cirrhosis and ascites, two were active smokers, one had a history of intravenous drug abuse, and three had a significant Morel-Lavallée lesion (Figure 2). One patient had a prior supraacetabular external fixator for two days, but different holes were used for the INFIX. (Figure 3 case example). Due to the small number of patients, we could not attribute any significantly increased risk from these variables compared to the noninfected cases.

Motor Vehicle Collision was the most common mechanism of injury (6/10), followed by fall from height (2/10), and pedestrian versus auto (2/10). All were closed injuries. There were two Young and Burgess LC1 injuries, two LC2 injuries, 


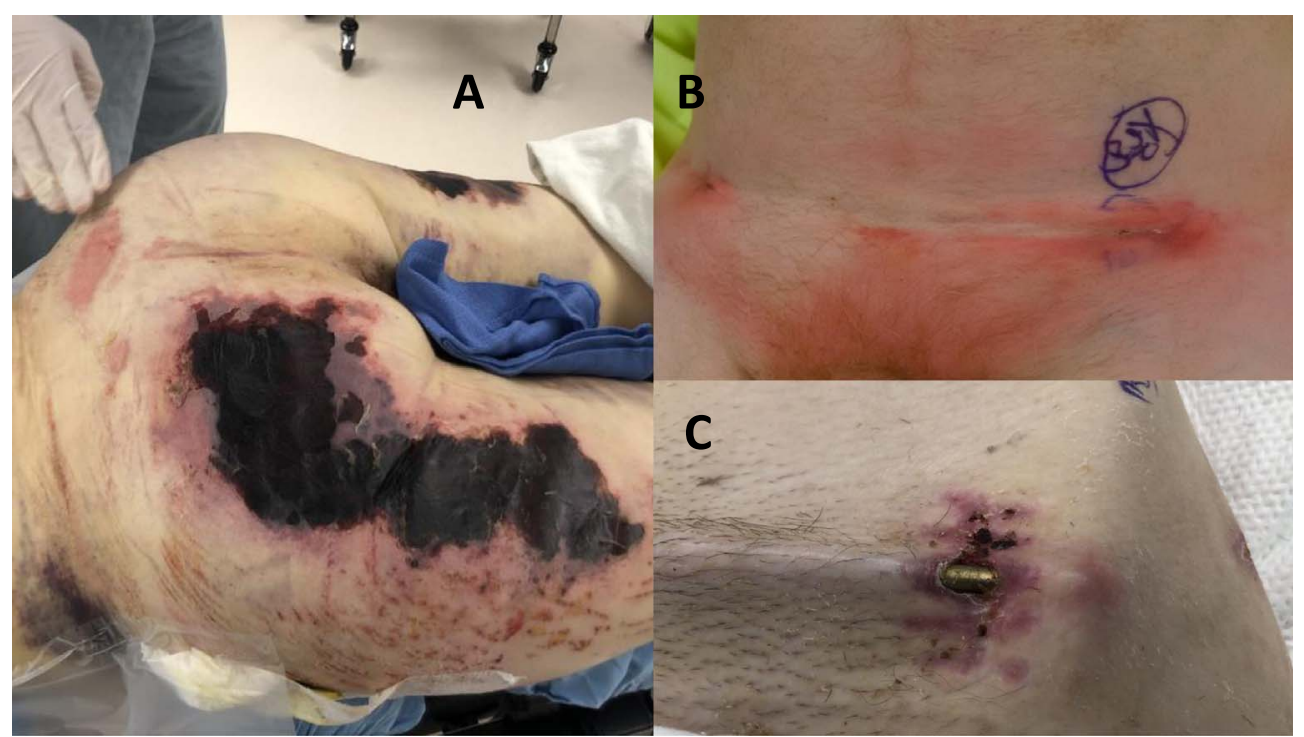

Figure 2. Clinical pictures of (a) a case of a large Morel-Lavallée lesion, (b) draining wound with exposed implants, (c) a draining wound and cellulitis.
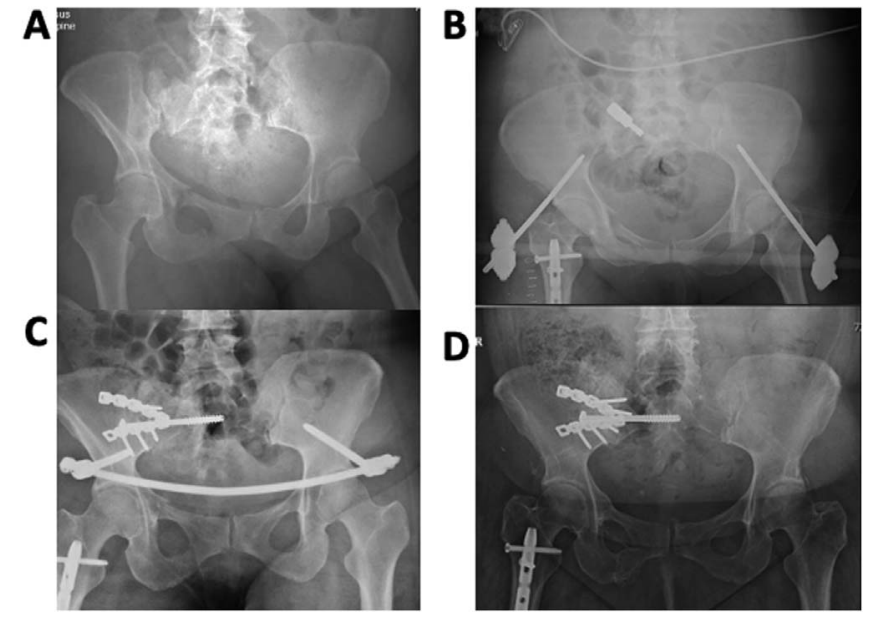

Figure 3. In one case, a supra-acetabular external fixator was used, and the resulting INFIX got infected despite using different holes for the implants. (a) Preop X-ray, (b) with temporary external fixator, (c) after definitive fixation, (d) at last follow-up.

three LC3 injuries, one APC2 injury, and two APC3 injuries (seven lateral compression injuries vs. three anterior posterior compression injuries).

Time to detect infection ranged from 24 to 90 days from the index procedure, with an average of $54.2 \pm 24.3$ days and a median of 56 days. In five cases, the infection was detected within 30 days, two cases between 30 and 60 days, and three cases between 60 and 90 days. In nine cases, the patient was taken to the OR within two days, and in one case, suppressive antibiotics were tried for 14 days which failed to suppress the drainage. The patient was then taken to the OR for formal I and $\mathrm{D}$ and removal of the implant.

Three patients had a second I and D procedure during this hospitalization and seven had a single procedure. The second procedure in three cases was that there was persistent drainage after the first washout.
There was no loss of reduction for any case due to explant as measured by the modified Keshishyan Index [24]. The earliest we removed implants was 25 days.

Staphylococcus aureus was the most common bacteria isolated and was found in $6 / 10$ patients. The other species identified included Enterobacter cloacae in two patients, Staphylococcus epidermidis in one, and Streptococcus agalactiae in the final patient (Table 2).

All patients had an infectious disease consult who recommended a culture-specific antibiotic regimen (Table 2) that continued as an out-patient for two weeks in seven patients, four weeks in one patient, and six weeks in two patients. This was based on the Infectious Disease Physicians recommendation, and unfortunately, it led to a varied duration of treatment. The length of stay for these patients due to the infection was 7.5 days \pm 5.4 range $1-18$ days in nine patients. One patient had a three-month length stay from his original injury unrelated to the pelvic fracture or INFIX infection. The infection was treated during his extended stay for abdominal injuries.

All the patients went on to radiographic union as determined by radiographs obtained in clinic and the ability to weight bear as tolerated with minimal pain. None of the patients developed recurrent infections at the INFIX site or any sign of osteomyelitis thus far.

Total Majeed scores for the patients ranged from 72 to 96 , with an average score of 81.7 at the latest follow-up. When converting these raw scores to functional status, all ten patients had final grading of good or excellent (seven rated as good and three as excellent).

\section{Discussion}

The use of INFIX in pelvic ring injuries has been adopted and reported from many centers globally [20]. What to do when an infection with this procedure has not been well delineated as it is uncommon (3\%) [20]. The rate of INFIX infection 
Table 2. Infection isolation and treatment data.

\begin{tabular}{|c|c|c|c|c|}
\hline & Bug isolated & Hospital Abx & Discharge Abx & Out pt tx \\
\hline 1 & Strep. agalactiae & Vancomycin iv & Clindamycin po & 6 weeks \\
\hline 2 & MSSA & $\begin{array}{l}\text { Sulfamethoxazole \& trimethoprim } \\
\text { double strength po }\end{array}$ & $\begin{array}{l}\text { Sulfamethoxazole \& trimethoprim } \\
\text { double strength po }\end{array}$ & 2 weeks \\
\hline 3 & Enterobacter cloacae & Ertapenem iv & Ciprofloxacin po & 2 weeks \\
\hline 4 & MSSA & Ceftriaxone iv & Clindamycin po & 4 weeks \\
\hline 5 & $\begin{array}{l}\text { Corynebacterium, Staphylococcus } \\
\text { epidermidis, Proteus mirabilis }\end{array}$ & Clindamycin iv & Clindamycin po & 2 weeks \\
\hline 6 & MRSA & Vancomycin iv & Vancomycin iv & 6 weeks \\
\hline 7 & MSSA & Vancomycin iv & $\begin{array}{l}\text { Sulfamethoxazole \& trimethoprim } \\
\text { double strength po }\end{array}$ & 2 weeks \\
\hline 8 & MRSA & Vancomycin iv & $\begin{array}{l}\text { Sulfamethoxazole \& trimethoprim } \\
\text { double strength po }\end{array}$ & 2 weeks \\
\hline 9 & Enterobacter cloacae & Ertapenem iv & Ciprofloxacin po & 2 weeks \\
\hline 10 & MSSA & Cefazolin iv & Cefazolin iv & 2 weeks \\
\hline
\end{tabular}

MRSA, methicillin-resistant Staphylococcus aureus; MSSA, methicillin sensitive Staphylococcus aureus; iv, intravenous; po, oral (par os).

Table 3. Infix infection literature review.

\begin{tabular}{|c|c|c|c|c|c|c|}
\hline Infection & Number & Early, $<10$ wks & Late & Explant & Kept & Total cases \\
\hline Vaidya et al. $[3,4]$ & 3 & 3 & & 3 & & 91 \\
\hline Merriman et al. [6] & 1 & & 1 & 1 & & 3 \\
\hline Müller et al. [7] & 2 & 2 & & 1 & 1 & 36 \\
\hline Hoskins et al. [10] & 3 & NR & & NR & NR & 21 \\
\hline Fang et al. [12] & 1 & 1 & & 1 & & 29 \\
\hline Shetty et al. [17] & 1 & 1 & & & 1 & 15 \\
\hline Dahill et al. [18] & 1 & & 1 & 1 & & 47 \\
\hline Steer et al. [20] & 2 & 2 & & 1 & 1 & 24 \\
\hline Current Study & 10 & 7 & 3 & 10 & & 179 \\
\hline Total & 24 & 16 & 5 & 18 & 3 & 445 \\
\hline
\end{tabular}

in this series was $5.6 \%(10 / 179)$. The diagnosis was made between three weeks and three months, it was based on drainage from the wounds and was confirmed with positive cultures. Risk factors included diabetes (1), liver cirrhosis (1), smoking (2), IV drug abuser (1), and a significant Morel-Lavallée lesion (3). We could not establish any significant associations or risk factors with these low numbers compared to the overall group. One patient had a prior supraacetabular external fixator for two days, and when we placed the INFIX, we drilled new holes for the pedicle screws, but this did not prevent an infection from occurring. In our practice, if we are placing an emergency anterior external fixator, we try to use iliac crest pins so that if we use an INFIX for definitive fixation, the supra acetabular sites are clean. All patients had the implant removed at 25 days or later post-surgery, and no loss of reduction was noted. Seven patients had a single washout following our protocol, and three had two washouts related to persistent drainage from the wound. We used a Yankauer tip to thoroughly irrigate the subcutaneous tract and the supraacetabular pin sites, which were also curetted. This seems to work, but there is no comparison procedure as this was what we did, and it was not based on prior knowledge in these cases. Antibiotics were given based on the organism isolated. Intravenous antibiotics were given in the hospital, and then outpatient antibiotics were prescribed for two weeks (seven patients), four weeks (one patient), and six weeks (two patients) after discharge based on the Infectious Disease team's recommendation. That did not make any difference in the long-term outcome. No patient suffered a second infection after removal of the implants, and there was no instance of osteomyelitis during the follow-up period, which averaged $830 \pm 170$ days. All these pelvic fractures healed. This was based on X-rays which showed healing and the ability to weight bear without difficulty or loss of reduction.

The limitations of this study and topic are that there are only ten cases of INFIX infection in this study and only 14 others in the literature, so it is difficult to make solid recommendations. This is simply a description of what was done and the outcome.

In a recent systemic review on the use of INFIX in pelvic injuries, infection at the surgical site was reported in 15 of 496 cases (3\%) [19]. In 9/15 cases, the infection occurred before 10 weeks, and in 3/15 cases, it occurred late after six months (three cases not elaborated). The implant was removed in ten cases, followed by culture-specific antibiotics. In two early cases, the infection was treated with culture-specific suppressive antibiotics, and it resolved to allow for explantation at the usual time. Infections resolved in all cases with no reports of osteomyelitis at the latest follow-up (Table 3).

In the current series, antibiotic suppression of the infection was attempted in one case to delay the removal of the implant. This did not work as there was continued drainage, 
and eventually, the hardware was removed two weeks later. The patient tolerated the draining wound.

From these cases and review of the literature, it seems that having the INFIX in for at least four weeks is adequate to maintain the overall reduction in the face of infection. We have also wondered that if the INFIX is exposed, is an absolute indication for removal. In several cases with massive pelvic wounds and soft tissue coverage issues, the INFIX has been utilized as a low elevation external fixator or exposed on one side while being covered on the opposite side. Due to its low profile, it is easy to cover the implant, and the wounds with a wound vacuum-assisted closure device (vac). In these cases, this also did not lead to osteomyelitis and was tolerated by the patients [25]. There have been reports of using suppressive antibiotics in the face of INFIX infection in several patients until the 3-month mark, and we feel that probably four weeks is fadequate to remove the implant as most pelvic fractures get sticky at this time [19]. We had no loss of reduction when the infix was removed after 25 days.

\section{Conclusion}

The primary method of treatment of infections after using the INFIX involved irrigating and debriding the wounds, removing the INFIX implant after a minimum of four weeks and treating with 2-4 weeks of culture-specific antibiotics. In early infections ( $<4$ weeks, two reported in the literature), the choice seems to be to irrigate, debride, treat with suppressive antibiotics and hopefully remove the implant after four weeks. There were no long-term sequelae that were noted in this series or the literature review.

\section{Conflicts of interest}

RV owns patents on Anterior Subcutaneous Pelvic Fixation / the INFIX Procedure, which have never restricted any use of the procedure and are available for use. The remaining authors have no relevant conflicts of interest to declare that could jeopardize the validity of the content of this article.

\section{Funding}

No funding was received to assist with the preparation of this manuscript.

\section{Ethics approval}

The study was approved by the IRB of our home institution.

\section{Informed consent}

Written or verbal informed consent was obtained from all patients included in the study.

\section{Authors' contributions}

K. Amar and D. Woodbury: data gathering; R. Vaidya: drafting the manuscript, data presentation, and data gathering; A. Washington: manuscript preparation, formatting, and editing.

\section{Supplementary Material}

Supplementary material is available at https://www.sicot-j. org/10.1051/sicotj/2021047/olm.

Video S1: Using the Yankauer tip to irrigate the subcutaneous tunnel.

Acknowledgements. Authors have no acknowledgments they wish to include.

\section{References}

1. Parsell DE, Cole PA. Device and method for less invasive stabilization of pelvic fractures. U.S. Patent 8,398,637 filed October 5, 2007, and issued March 19, 2013.

2. Vaidya R (2012) Method for minimally invasive treatment of unstable pelvic ring injuries with an internal anterior fixator and posterior iliosacral screws. 8,177,785 filed October 9, 2008 and issued May 152012.

3. Vaidya R, Colen R, Vigdorchik J, Tonnos F, Sethi A (2012) Treatment of unstable pelvic ring injuries with an internal anterior fixator and posterior fixation: initial clinical series. J Orthop Trauma 26(1), 1-8.

4. Vaidya R, Kubiak EN, Bergin PF, Dombroski DG, Critchlow RJ, Sethi A, Starr AJ (2012) Complications of anterior subcutaneous internal fixation for unstable pelvis fractures: a multicenter study. Clin Orthop Relat Res 470(8), 2124-2131.

5. Gardner MJ, Mehta S, Mirza A, Ricci WM (2012) Anterior pelvic reduction and fixation using a subcutaneous internal fixator. J Orthop Trauma 26(5), 314-321.

6. Merriman DJ, Ricci WM, McAndrew CM, Gardner MJ (2012) Is application of an internal anterior pelvic fixator anatomically feasible? Clin Orthop Relat Res 470(8), 2111-2115.

7. Müller FJ, Stosiek W, Zellner M, Neugebauer R, Füchtmeier B (2013) The anterior subcutaneous internal fixator (ASIF) for unstable pelvic ring fractures: clinical and radiological mid-term results. Int Orthop 37(11), 2239-2245.

8. Owen MT, Tinkler B, Stewart R (2013) Failure and salvage of "INFIX" instrumentation for pelvic ring disruption in a morbidly obese patient. J Orthop Trauma 27(10), e243-6. Erratum in: J Orthop Trauma. 2014;28(3):180.

9. Hesse D, Kandmir U, Solberg B, Stroh A, Osgood G, Sems SA, Collinge CA (2015) Femoral nerve palsy after pelvic fracture treated with INFIX: a case series. J Orthop Trauma 29(3), 138-143.

10. Hoskins W, Bucknill A, Wong J, Britton E, Judson R, Gumm K, Santos R, Sheehy R, Griffin X (2016) A prospective case series for a minimally invasive internal fixation device for anterior pelvic ring fractures. J Orthop Surg Res 11(1), 135.

11. Zhang Y, Liu Y, Wu C, et al. (2016) Treatment of unstable pelvic ring injuries with an internal anterior fixator: initial clinical series. Int J Clin Exp Med 9(9), 18637-18644.

12. Fang C, Alabdulrahman H, Pape HC (2017) Complications after percutaneous internal fixator for anterior pelvic ring injuries. Int Orthop 41(9), 1785-1790. 
13. Smith A, Malek IA, Lewis J, Mohanty K (2017) Vascular occlusion following application of subcutaneous anterior pelvic fixation (INFIX) technique. J Orthop Surg (Hong Kong) 25(1), 2309499016684994.

14. Bi C, Wang Q, Wu J, Zhou F, Zhang F, Liang H, Lyu F, Wang J (2017) Modified pedicle screw-rod fixation versus anterior pelvic external fixation for the management of anterior pelvic ring fractures: a comparative study. J Orthop Surg Res 12(1), 185.

15. Vaidya R, Martin AJ, Roth M, Tonnos F, Oliphant B, Carlson J (2017) Midterm radiographic and functional outcomes of the anterior subcutaneous Internal Pelvic Fixator (INFIX) for pelvic ring injuries. J Orthop Trauma 31(5), 252-259.

16. Wang Q, Wang Q, Wang J (2017) Treatment of type B pelvic fracture using anterior subcutaneous internal fixator with triple pedicle screws: a new surgical technique. Arch Orthop Trauma Surg 137(7), 887-893.

17. Shetty AP, Bosco A, Perumal R, Dheenadhayalan J, Rajasekaran S (2017) Midterm radiologic and functional outcomes of minimally-invasive fixation of unstable pelvic fractures using anterior internal fixator (INFIX) and percutaneous iliosacral screws. J Clin Orthop Trauma 8(3), 241-248.

18. Dahill M, McArthur J, Roberts GL, Acharya MR, Ward AJ, Chesser TJS (2017) The use of an anterior pelvic internal fixator to treat disruptions of the anterior pelvic ring: a report of technique, indications and complications. Bone Joint J 99(9), 1232-1236.
19. Vaidya R, Woodbury D, Nasr K (2018) Anterior subcutaneous Internal Pelvic Fixation/INFIX: a systemic review. J Orthop Trauma 32(Suppl 6), S24-S30.

20. Steer R, Balendra G, Matthews J, Wullschleger M, Reidy J (2019) The use of anterior subcutaneous internal fixation (INFIX) for treatment of pelvic ring injuries in major trauma patients, complications and outcomes. SICOT J 5, 22.

21. Burgess AR, Eastridge BJ, Young JW, Ellison TS, Ellison PS Jr, Poka A, Bathon GH, Brumback RJ (1990) Pelvic ring disruptions: effective classification system and treatment protocols. J Trauma 30(7), 848-856.

22. Majeed SA (1990) External fixation of the injured pelvis. The functional outcome. J Bone Joint Surg Br 72(4), 612-614.

23. Keshishyan RA, Rozinov VM, Malakhov OA, Kuznetsov LE, Strunin EG, Chogovadze GA, Tsukanov VE (1995) Pelvic polyfractures in children. Radiographic diagnosis and treatment. Clin Orthop Relat Res 320, 28-33.

24. Lefaivre KA, Blachut PA, Starr AJ, Slobogean GP, O'Brien PJ (2014) Radiographic displacement in pelvic ring disruption: reliability of 3 previously described measurement techniques. J Orthop Trauma 28(3), 160-166.

25. Vaidya R, Nasr K, Feria-Arias E, Fisher R, Kajy M, Diebel LN (2016) INFIX/EXFIX: massive open pelvic injuries and review of the literature. Case Rep Orthop 2016, 9468285.

Cite this article as: Vaidya R, Amar K, Woodbury D \& Washington A (2021) Infection after the use of INFIX in Pelvic Ring Injuries. SICOT-J 7, 46 